\title{
Atenuação da radiação incidente em compósito de ferrita de NiZn dopado com magnésio e cobre em epóxi
}

\section{(Attenuation of incident radiation in composite of NiZn ferrite doped with magnesium and copper in epoxy)}

\author{
R. C. Pessoa ${ }^{1}$, M. C. Nasar ${ }^{1}$, R. S. Nasar ${ }^{1}$, M. C. Rezende' , M. K. Hwang ${ }^{2}$ \\ ${ }^{1}$ Instituto de Química, UFRN,Campus Universitário, Lagoa Nova, Natal, RN, 59078-970 \\ ${ }^{2}$ Departamento de Ciência e Tecnologia Aeroespacial, Divisão de Materiais , Av. Brig. Faria Lima 1941, \\ IAE, S. J. Campos, SP 12228-904 \\ regiachacon@ig.com.br,mari.nat30@uol.com.br,nasar@terra.com.br, \\ mirabelrezende@uol.com.br,miriamk@cta.br
}

\begin{abstract}
Resumo
Foi sintetizada uma ferrita espinélio de $\mathrm{NiZnFe}_{2} \mathrm{O}_{4}$ dopada com magnésio e cobre pelo método dos citratos precursores, para pesquisar a influência desses íons na refletividade do pó. Os pós calcinados a 350,500 e $900{ }^{\circ} \mathrm{C}$ foram caracterizados por difração de raios X, microscopia eletrônica de varredura, magnetômetro de amostra vibrante e refletividade do pó. O pó obtido a $350{ }^{\circ} \mathrm{C}$ é nanométrico e apresentou baixa magnetização e menos de $10 \%$ de absorção de radiação. A magnetização e a absorção de radiação aumentaram com o aumento do tamanho de partículas $(>0,4 \mu \mathrm{m})$, que causou aumento do volume dos domínios magnéticos. O pó calcinado a $900{ }^{\circ} \mathrm{C} / 3 \mathrm{~h}$ obteve atenuação máxima de $-6,4 \mathrm{~dB}$ (77 \% de absorção de radiação) abrangendo a região de microondas de $8,2 \mathrm{GHz}$ e diminuindo na faixa da banda X. Esses materiais magnéticos que absorvem acima de $35 \%$ de radiação na faixa de microondas podem ser aplicados no desenvolvimento de antenas para telefonia celular.
\end{abstract}

Palavras-chave: MARE, materiais magnéticos, refletividade.

\section{Abstract}

$A \mathrm{NiZnFe}_{2} \mathrm{O}_{4}$ spinel ferrite doped with magnesium and copper was synthesized by the citrate precursor method to explore the influence of these ions in the reflectivity of the powder. The powders calcined at 350, 500 and $900{ }^{\circ} \mathrm{C}$ were characterized by $X$-ray diffraction, scanning electron microscopy, vibrating sample magnetometer and reflectivity. The powder obtained at $350{ }^{\circ} \mathrm{C}$ is nanosized and showed low magnetization and less than 10\% absorption of radiation. The magnetization and radiation absorption increase with increasing particle size $(>0.4 \mu \mathrm{m})$, causing an increase in volume of the magnetic domains. The powder calcined at $900{ }^{\circ} \mathrm{C} / 3 \mathrm{~h}$ reached maximum attenuation of $-6.4 \mathrm{~dB}$ (77\% absorption of radiation) covering the region of $8.2 \mathrm{GHz}$ microwave and decreasing in the range of the $X$ band. These magnetic materials that absorb over $35 \%$ of radiation in the microwave range can be applied to the development of antennas for cellular telephony.

Keywords: RAM, magnetic materials, reflectivity.

\section{INTRODUÇÃO}

Devido ao rápido desenvolvimento da nanotecnologia e a busca por novos materiais com alto desempenho, as ferrites se tornaram um dos principais focos das atividades de pesquisa, desenvolvimento e inovação em países industrializados. Materiais ferrimagnéticos à base de $\mathrm{NiZnFe}_{2} \mathrm{O}_{4}$ são objeto de pesquisas devido à grande quantidade de aplicativos na área científica e principalmente tecnológica [1]. Aditivos tais como cobre e magnésio melhoram as características magnéticas desses materiais. Atualmente várias pesquisas são realizadas no sentido da aplicação de ferritas de NiZn em materiais absorvedores de radiação eletromagnética, MARE, para emprego na área de telefonia móvel (telefones celulares) [2]. Vários métodos de síntese são usados no desenvolvimento dos materiais e dentre estes o processo da síntese por citratos é de alto interesse tecnológico $[3,4]$. A adição de cobre na ferrita de NiZn substitui posições octaédricas na estrutura espinélio e aumenta a magnetização por cela unitária. No entanto, o estado final da magnetização depende das características das partículas após a calcinação ou da microestrutura formada após a sinterização. Na obtenção de pós finos foram desenvolvidas ferritas de NiZn dopadas com cobre usando o método da co-precipitação [5]. As composições variaram de $\mathrm{x}=0$ até $\mathrm{x}=0,6 \mathrm{em}$ mol de cobre substituindo níquel na estrutura espinélio. A difração de raios $\mathrm{X}$ mostrou a formação da fase espinélio até a concentração de $0,25 \mathrm{~mol} \%$ de cobre. Foi observada máxima coercitividade quando o tamanho médio da partícula foi próximo do diâmetro crítico magnético do cristal. Outra pesquisa, usando o método da coprecipitação obteve pós ultra finos e foi observado por 
difração de raios X que acima da concentração de 0,42 mol\% de cobre ocorre a formação das fases espinélio e óxido de cobre [6]. Conclusivamente, ambos resultados mostram que somente pequenas concentrações de cobre favorecem a magnetização do sistema NiZn. Ferritas de niquel-cobrezinco foram sintetizadas pelo método dos citratos, obtendo pós nanométricos em baixa temperatura com fase única tipo espinélio $[7,8]$. A caracterização do material quanto à absorção de radiação obteve $96,6 \%$ de refletividade na faixa de 8-10 GHz de freqüência.

Neste trabalho foi desenvolvida uma ferrita de níquelzinco-magnésio-cobre pelo método dos citratos. O objetivo foi verificar qual a contribuição da adição de cobre e magnésio, considerando que $\mathrm{o} \mathrm{Mg}$ em princípio não contribui para o aumento da magnetização do material, nas características magnéticas e características de absorção de radiação eletromagnética dos pós de níquel-zinco-magnésiocobre. Não foi reportada pesquisa comparativa considerando material magnético de níquel-zinco com adições simultâneas de magnésio e cobre aplicadas para material absorvedor de radiação eletromagnética.

\section{MATERIAIS E MÉTODOS}

Os reagentes químicos de alta pureza usados na síntese dos pós estão descritos na Tabela I. Todos os reagentes são de grau analítico para a preparação dos citratos dos metais de níquel, zinco, ferro, cobre e magnésio.

Tabela I - Reagentes utilizados na síntese dos materiais. [Table I - Reagents used in the materials synthesis.]

\begin{tabular}{ccc}
\hline Reagentes & Pureza & Origem \\
\hline Ácido cítrico & $99,50 \%$ & Vetec \\
Nitrato de ferro monohidratado & $99,00 \%$ & Aldrich \\
Nitrato de zinco hexahidratado & $99,00 \%$ & Aldrich \\
Nitrato de níquel hexahidratado & $99,99 \%$ & Aldrich \\
Nitrato de magnésio hexahidratado & $99,00 \%$ & Aldrich \\
$\begin{array}{c}\text { Nitrato de cobre II } \\
\text { hemipentahidratado }\end{array}$ & $99,99 \%$ & Aldrich \\
\hline
\end{tabular}

Na preparação dos citratos, o ácido cítrico foi misturado na proporção de $1 \mathrm{~mol}$ de ácido para $2 \mathrm{~mol}$ de nitrato para todos os reagentes. As soluções foram misturadas separadamente e homogeneizadas durante $1 \mathrm{~h}$ e então ocorreu uma reação de complexação próximo de $90{ }^{\circ} \mathrm{C}$. Os citratos foram misturados para formar a seguinte solução sólida: $\mathrm{Ni}_{0,12} \mathrm{Zn}_{0,50} \mathrm{Mg}_{0,08} \mathrm{Cu}_{0,30} \mathrm{Fe}_{2} \mathrm{O}_{4}$. Os citratos de níquel, zinco, ferro, magnésio e cobre foram misturados e homogeneizados por agitação magnética durante $2 \mathrm{~h}$. A solução orgânica de citratos foi então decomposta em um forno tipo mufla a $350{ }^{\circ} \mathrm{C}$ durante $3 \mathrm{~h}$. O pó formado foi então desaglomerado em almofariz e peneirado em malha 325 ABNT. O pó foi caracterizado por difração de raios X (DRX) num difratômetro Shimadzu XRD 6000 com fonte Cuk $\alpha(1,5418 \AA)$ com tensão $30 \mathrm{kV}$ e corrente $20 \mathrm{~mA} \mathrm{e}$ também por magnetometria de amostra vibrante (MAV). Este equipamento permite medir magnetização entre $77 \mathrm{e}$ $650 \mathrm{~K}$ em campos magnéticos até $10 \mathrm{kOe}$. Após calcinação a $350{ }^{\circ} \mathrm{C}$ e caracterização, o pó foi separado em dois lotes e então calcinado a $500{ }^{\circ} \mathrm{C} / 3 \mathrm{~h}$ e $900{ }^{\circ} \mathrm{C} / 3 \mathrm{~h}$ em atmosfera ambiente. Os pós foram caracterizados por DRX e MAV. Outros lotes de pós foram moídos e peneirados em malha 325 ABNT e então misturados nas proporções de $25 \%$ de pó para $75 \%$ de resina epóxi para confecção dos corpos de prova para medidas de refletividade. A refletividade foi medida por processo de guia de ondas por um acoplador direcional constituído de 2 guias de ondas acoplados. Os equipamentos foram: acoplador direcional modelo X752C Hewlett Packard, gerador de sinais modelo 83752A Agilent e carga de guia de onda Systron-Donner DBG-458. Os pós foram analisados por microscopia eletrônica de varredura (MEV) em um equipamento Philips XL30 ESEM com voltagem de aceleração $20 \mathrm{kV}$. O tamanho médio de partículas e as fases cerâmicas foram analisados por meio do método de Rietveld com o programa Maud versão 2.044.

\section{RESULTADOS E DISCUSSÃO}

Na Fig. 1 são observadas os difratogramas de raios $\mathrm{X}$ dos pós calcinados a 350,500 e $900{ }^{\circ} \mathrm{C} / 3 \mathrm{~h}$. Foi observada a formação de fase única do tipo espinélio cúbica a 350 e $500{ }^{\circ} \mathrm{C}$. O pó calcinado a $900{ }^{\circ} \mathrm{C}$ mostrou uma pequena porcentagem de fase de óxido de cobre e fase espinélio majoritária. O processo de difusão do magnésio e do cobre formou solução sólida homogênea nos pós calcinados a temperaturas baixas de calcinação. Em temperatura elevada, uma pequena parte do cobre na amostra formou (segregou) a fase de óxido de cobre pura.

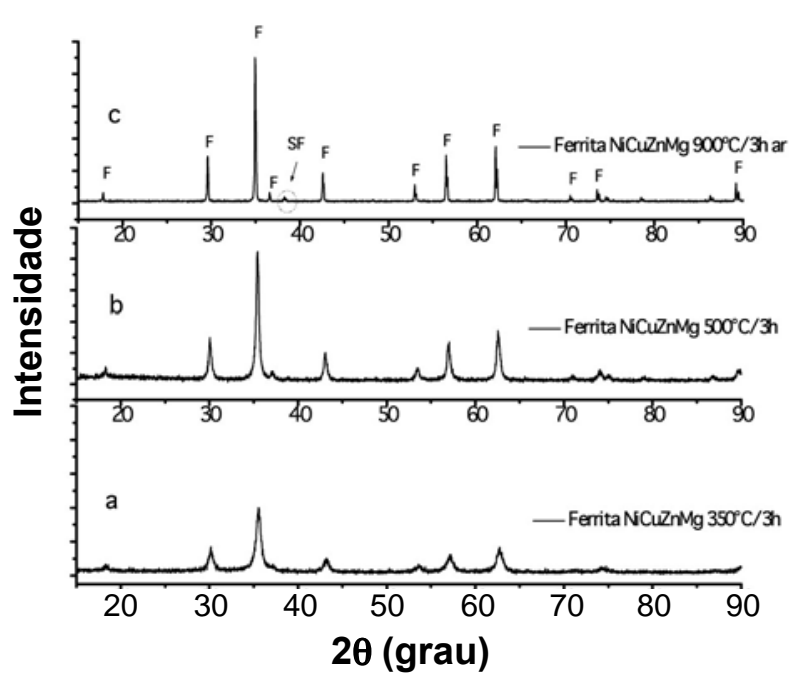

Figura 1: Difratogramas de raios $\mathrm{X}$ do $\mathrm{NiCuZnMg}$ calcinado a $350{ }^{\circ} \mathrm{C} / 3 \mathrm{~h} \mathrm{(a),} 500{ }^{\circ} \mathrm{C} / 3 \mathrm{~h}(\mathrm{~b})$ e a $900{ }^{\circ} \mathrm{C} / 3 \mathrm{~h} \mathrm{(c)}$.

[Figure 1: X-ray diffraction patterns of $\mathrm{NiCuZnMg}$ calcined at $350{ }^{\circ} \mathrm{C} / 3 \mathrm{~h}(\mathrm{a}), 500{ }^{\circ} \mathrm{C} / 3 \mathrm{~h}(\mathrm{~b})$ and $\left.900{ }^{\circ} \mathrm{C} / 3 \mathrm{~h}(\mathrm{c}).\right]$

Na Tabela II são mostradas as quantidades de fases obtidas pelo refinamento de Rietveld dos pós calcinados, 
Tabela II - Parâmetros de rede, tamanho de cristalito e quantidade de fases em função da temperatura de calcinação dos pós. [Table II - Lattice parameters, crystallite size and phase quantity as a function of calcining temperature of powder.]

\begin{tabular}{cccccccccc}
\hline Temperature $\left({ }^{\circ} \mathrm{C}\right)$ & Fase & $\mathrm{a}(\AA)$ & $\mathrm{Tc}(\mathrm{nm})$ & $\%$ Fase & $\operatorname{Rexp}$ & $R w$ & $R n w$ & Sig & $R a$ \\
\hline 350 & Ferrita & 8,37 & $13,40 \pm 1,65$ & 100 & 18,74 & 21,56 & 0,21 & 1,15 & 16,11 \\
500 & Ferrita & 8,38 & $29,76 \pm 1,22$ & 100 & 14,69 & 18,24 & 0,18 & 1,24 & 14,01 \\
900 & Ferrita & 8,39 & $977,76 \pm 59,42$ & 99,2 & 15,48 & 18,04 & 0,18 & 1,16 & 13,73 \\
& Óxido de cobre & & $100,03 \pm 11,32$ & 0,8 & & & & & \\
\hline
\end{tabular}

com $100 \%$ de fase ferrita a 350 e $500{ }^{\circ} \mathrm{C}$ e $99,2 \%$ de fase ferrita com $0,8 \%$ de fase de óxido de cobre a $900{ }^{\circ} \mathrm{C}$. No detalhe (SF) da difração de raios $\mathrm{X}$, considerando-se que foi formado menos de $1 \%$ da fase de óxido de cobre, a intensidade do pico $100 \%$ desta fase é muito pequena e os outros picos, de menores intensidades, na maioria sobrepostos, não são observados. Foi observada a formação de partículas nanométricas nas composições de fase única. Ocorreu o aumento dos parâmetros de rede da fase espinélio com o aumento da temperatura de calcinação. Isto indica que as substituições de magnésio e de cobre aumentaram o volume da cela unitária da estrutura.

Na Tabela III são comparados os níveis de ocupação de três ferritas, tais como: Ni Mg (B-I), NiCuZn (B-II) e $\mathrm{NiCuZnMg} \mathrm{(B-III).} \mathrm{No} \mathrm{caso} \mathrm{de} \mathrm{B-III} \mathrm{os} \mathrm{átomos} \mathrm{de} \mathrm{cobre} \mathrm{em}$ posições tetraédricas diminuem a concentração de íons $\mathrm{Fe}$ nas mesmas posições e aumenta o efeito de magnetização da cela unitária. A análise do material B-II mostra que os íons cobre substituem posições tetraédricas o que não favorece ao efeito de magnetização. As migrações de íons na estrutura modificam as características da magnetização resultante na estrutura espinélio. Na ferrita B-I o Mg induz a diminuição de íons Fe na posição octaédrica aumentando a concentração desses íons nas posições tetraédricas o que causa uma diminuição em termos da magnetização da cela unitária. No entanto fatores da microestrutura são fundamentais na magnetização resultante de cada sistema.

A análise dos pós por microscopia eletrônica, Fig. 2, mostrou que o tamanho médio de partículas é próximo dos obtidos pelo método de Rietveld. No pó calcinado a $350{ }^{\circ} \mathrm{C}$ as partículas apresentam tamanhos de $\sim 20$ a $50 \mathrm{~nm}$. O pó calcinado a $500{ }^{\circ} \mathrm{C}$ mostrou partículas entre 50 e $200 \mathrm{~nm}$ e o material calcinado a $900{ }^{\circ} \mathrm{C}$ mostrou pequenas partículas devidas à formação da fase óxido de cobre juntamente com partículas com $1 \mu \mathrm{m}$ de diâmetro da fase ferrita. Isto indica que o processamento cerâmico foi eficiente na obtenção de fase homogênea e única, tipo cúbica, em baixa temperatura de calcinação. A partícula obtida nanométrica cresce homogênea em forma e distribuição granulométrica estreita entre 500 e $600{ }^{\circ} \mathrm{C}$. A temperaturas mais altas ocorre segregação parcial do cobre formando a fase óxido de cobre.

A Fig. 3 mostra as histereses ferrimagnéticas dos pós calcinados a 350,500 e $900{ }^{\circ} \mathrm{C}$. Quanto maior o tamanho das partículas maior o efeito total da magnetização das ferritas. As partículas nanométricas do pó calcinado a 350 ${ }^{\circ} \mathrm{C}$ mostraram baixa magnetização total. Partículas com tamanhos nanométricos não apresentam estruturas de domínios magnéticos. O processo de magnetização depende da magnetização local (em cada átomo), contribuindo para uma resultante magnética residual. No entanto, partículas com tamanho aproximado de $400 \mathrm{~nm}$ apresentam monodomínios e acima deste tamanho de partículas podem ter multidomínios, o que contribui com o efeito total da magnetização. Isto fica evidente quando se analisa os valores de magnetização de saturação e magnetização remanescente na Tabela IV, na qual as partículas maiores (calcinada a $900{ }^{\circ} \mathrm{C}$ ) apresentaram $\mathrm{Ms}$ de $66,54 \mathrm{Am}^{2} / \mathrm{Kg}$ e as partículas pequenas (nanométricas) apresentaram $\mathrm{Ms}$ de 6,20 $\mathrm{Am}^{2} /$ $\mathrm{Kg}$ mostrando também que a magnetização remanescente é maior quanto maior a partícula. Isto indica que o momento

Tabela III - Parâmetros estruturais das amostras de composição B-I, B-II e B-III.

[Table III - Structural parameters of samples of B-I, B-II and B-III composition.]

\begin{tabular}{ccccccc}
\hline Átomos & \multicolumn{3}{c}{ Posições } & \multicolumn{3}{c}{ Níveis de ocupação } \\
\hline & $\mathrm{x}$ & $\mathrm{y}$ & $\mathrm{Z}$ & $\mathrm{B}-\mathrm{I}$ & $\mathrm{B}-\mathrm{II}$ & $\mathrm{B}-\mathrm{III}$ \\
$\mathrm{Fe}$ (octaédrica) & 0,62 & 0,62 & 0,62 & 0,590 & 0,750 & 0,765 \\
$\mathrm{Fe}$ (tetraédrica) & 0 & 0 & 0 & 0,820 & 0,500 & 0,470 \\
$\mathrm{Mg}$ (octaédrica) & 0,62 & 0,62 & 0,62 & 0,160 & - & 0,025 \\
$\mathrm{Mg}$ (tetraédrica) & 0 & 0 & 0 & 0,180 & - & 0,030 \\
$\mathrm{Zn}$ (tetraédrica) & 0 & 0 & 0 & - & 0,500 & 0,500 \\
Ni(octaédrica) & 0,62 & 0,62 & 0,62 & 0,250 & 0,100 & 0,060 \\
$\mathrm{Cu}$ (octaédrica) & 0,62 & 0,62 & 0,62 & - & 0,150 & 0,140 \\
$\mathrm{Cu}$ (tetraédrica) & 0 & 0 & 0 & & - & 0,020 \\
\hline
\end{tabular}



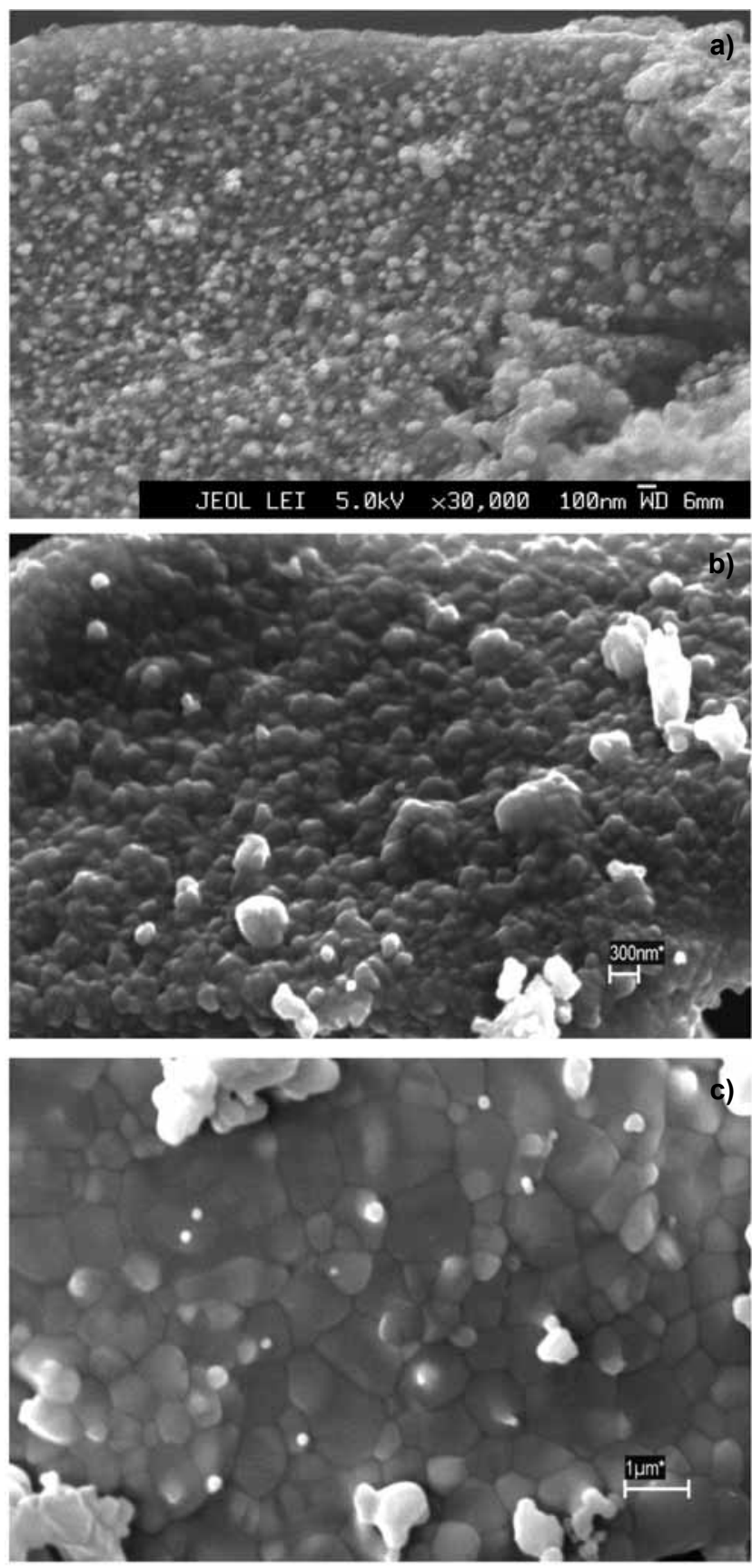

Figura 2: Pós de ferritas calcinadas em atmosfera inerte. Pó calcinado a $350{ }^{\circ} \mathrm{C} / 3 \mathrm{~h}$ (a), $500{ }^{\circ} \mathrm{C} / 3 \mathrm{~h} \mathrm{(b),} 900{ }^{\circ} \mathrm{C} / 3 \mathrm{~h}$ (c).

[Figure 2: Ferrite powders calcined in inert atmosfere. Powder calcined at $350{ }^{\circ} \mathrm{C} / 3 \mathrm{~h}(\mathrm{a}), 500{ }^{\circ} \mathrm{C} / 3 \mathrm{~h}(\mathrm{~b}), 900{ }^{\circ} \mathrm{C} / 3 \mathrm{~h}(\mathrm{c})$.]

magnético por domínio é maior nas partículas maiores.

No sistema níquel-zinco-magnésio-cobre observa-se que a adição de cobre pode causar a formação de vacâncias de oxigênio devido a alterações do número de oxidação do cobre. As vacâncias em concentrações moderadas aliviam o campo de tensão mecânica nos limites dos domínios e favorecem a expansão dos domínios magnéticos devido a instabilidade do campo magnético próximo das vacâncias. Isto explica sistemas com baixa concentração de ions cobre que causam o aumento da magnetização total. $\mathrm{O}$ aumento de um elétron em átomos de cobre em posição octaédrica

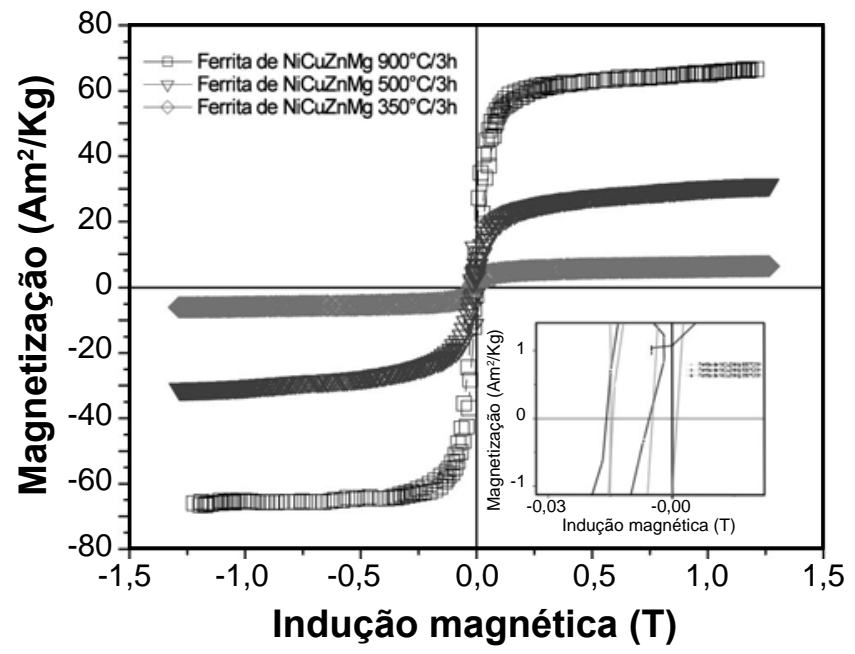

Figura 3: Magnetização de saturação versus indução magnética (histerese magnética).

[Figure 3: Saturation magnetization versus magnetic induction (magnetic hystheresis).]

Tabela IV - Magnetização de saturação $\mathrm{M}_{\mathrm{S}}$, magnetização remanescente $\mathrm{M}_{\mathrm{R}}$ e campo coercitivo $\mathrm{H}_{\mathrm{C}}$.

[Table IV - Saturation magnetization $M_{S}$, remnant magnetization $M_{R}$ and coercive field $H_{C}$ ]

\begin{tabular}{cccc}
\hline $\begin{array}{c}\text { Temperatura } \\
\left({ }^{\circ} \mathrm{C}\right)\end{array}$ & $\begin{array}{c}\mathrm{M}_{\mathrm{S}} \\
\left(\mathrm{Am}^{2} / \mathrm{kg}\right)\end{array}$ & $\begin{array}{c}\mathrm{M}_{\mathrm{R}} \\
\left(\mathrm{Am}^{2} / \mathrm{kg}\right)\end{array}$ & $\begin{array}{c}\mathrm{H}_{\mathrm{C}} \\
(\mathrm{T})\end{array}$ \\
\hline 350 & 6,20 & 0,89 & 0,005 \\
500 & 30,78 & 10,72 & 0,007 \\
900 & 66,54 & 11,58 & 0,009 \\
\hline
\end{tabular}

na estrutura espinélio causa uma diminuição no magneton de Bohr resultante na cela unitária. No entanto não se descarta a possibilidade dos átomos de cobre substituirem posições tetraédricas o que forçaria os íons de ferro migrarem para posições octaédricas aumentando assim o efeito geral da magnetização. Essa migração dos íons de cobre na estrutura espinélio pode ser observada na análise por Rietveld onde verifica-se que ocorre substituições de íons cobre tanto nas posições octaedrais quanto nas posições tetraedrais da estrutura. Isto explica o efeito do aumento da magnetização quando existe baixa concentração de cobre na estrutura e diminuição da magnetização total quando ocorre alta concentração de íons cobre no material. A adição de magnésio não deveria alterar as características magnéticas considerando que os spins da camada de valência estão emparelhados. Considerando que o raio atômico do magnésio é pequeno o íon pode substituir principalmente posições octaedrais bem como posições tetraedrais. O magnésio em posições octaedrais aumenta o efeito da magnetização na cela unitária devido a resultante da magnetização ser maior e o efeito é contrário quando em posições tetraedrais. Isto indica que a magnetização variará conforme as posições ocupadas por íons cobre e magnésio na cela unitária. Os valores de magneton de Bohr farão contribuições diferentes para o efeito final da magnetização. Deve-se verificar que. 


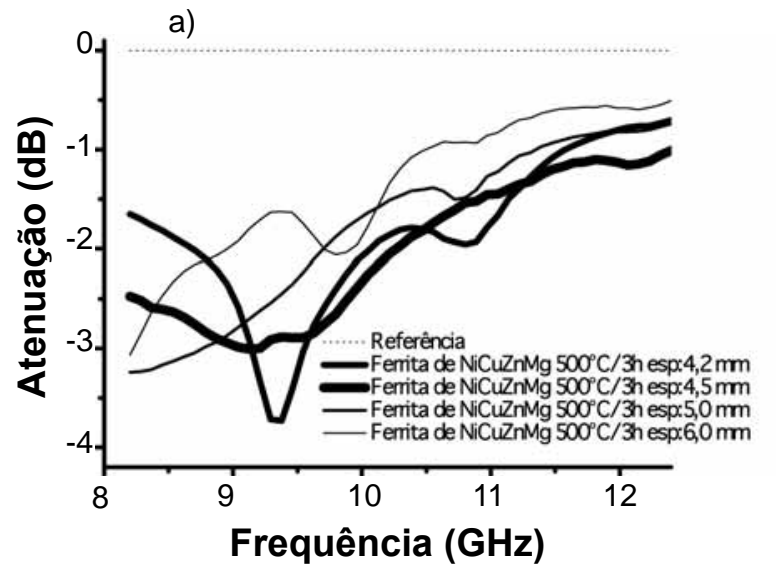

b)

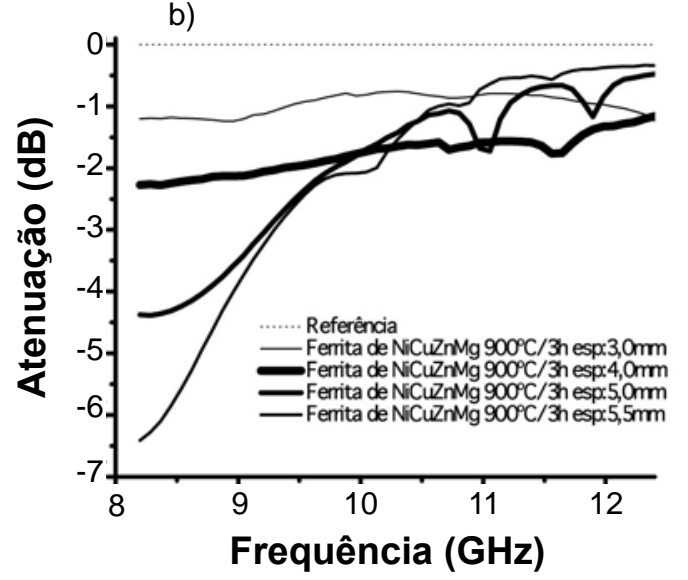

Figura 4: Medidas de refletividade dos compósitos. Pó calcinado a $500{ }^{\circ} \mathrm{C} / 3 \mathrm{~h} \mathrm{(a),} 900{ }^{\circ} \mathrm{C} / 3 \mathrm{~h}$ (b).

[Figure 4. Reflectivity measures of composites. Powder calcined at $\left.500{ }^{\circ} \mathrm{C} / 3 \mathrm{~h}(\mathrm{a}), 900{ }^{\circ} \mathrm{C} / 3 \mathrm{~h}(\mathrm{~b}).\right]$

de acordo com o nível de ocupação dos íons níquel, zinco e ferro no estudo da ferrita de NiZn [7], esses íons ocupam posições preferencialmente octaedrais na estrutura espinélio. A adição de cobre neste sistema substitui parcialmente íons de níquel na estrutura em posições octaedrais [8]. Isto corrobora com o fato da adição de cobre aumentar o efeito da magnetização no sistema NiZn. Como observado na Tabela III os íons magnésio e íons cobre substituem posições octaedrais, sendo que, o magnésio não deveria contribuir diretamente com o aumento do efeito magnético porque não altera o magneton de Bohr.

A medida de refletividade do pó calcinado a $350{ }^{\circ} \mathrm{C}$ não obteve resultado superior a $7 \%$ de absorção de radiação. A medida de histerese magnética mostrou baixa magnetização de saturação. As partículas nanométricas não apresentam estruturas de domínios e sim um comportamento de rotação coerente, o que explica a baixa magnetização e a capacidade muito limitada de absorção de radiação.

A Fig. 4 mostra as medidas de refletividade dos pós calcinados a $500{ }^{\circ} \mathrm{C}$ e $900{ }^{\circ} \mathrm{C}$. As medidas de refletividade obtidas a $500{ }^{\circ} \mathrm{C}$ são menores que as observadas na maior temperatura. Isto coincide com as medidas de histerese magnética que apresentam maior magnetização a $900^{\circ} \mathrm{C}$. Na temperatura menor os domínios magnéticos são menores, por volta de 100 a $200 \mathrm{~nm}$, e formam monodomínios o que causa menor absorção de radiação considerando a menor quantidade de spins orientados que contribuem favoravelmente à resultante magnética nos cristais. Nas curvas de refletividade da amostra calcinada a $500{ }^{\circ} \mathrm{C} / 3$ h observa-se uma atenuação máxima de $-3,7 \mathrm{~dB}(57,3 \%)$ mostrando um perfil de absorção em "V" com uma banda larga, atenuando na região de 9,3 a 9,4 GHz. Para a ferrita calcinada a $900{ }^{\circ} \mathrm{C} / 3 \mathrm{~h}$ é observado uma atenuação máxima de $-6,4 \mathrm{~dB}$, cerca de $77 \%$ de absorção da radiação incidente abrangendo a região de $8,2 \mathrm{GHz}$, diminuindo ao longo da banda X. Verifica-se que a espessura do compósito influencia a refletividade, considerando que quanto maior o tamanho do cristal maior deve ser a espessura do compósito. Os cristais maiores apresentam estrutura de multidomínios magnéticos resultando em maior magnetização e causando maior absorção de radiação eletromagnética.

Materiais que absorvem acima de $35 \%$ de radiação na faixa de microondas são aplicados no desenvolvimento de antenas para telefonia celular. $\mathrm{O}$ aprimoramento dos materiais de absorção de radiação aumentam a eficiência dos emissores e receptores de microondas destes dispositivos.

\section{CONCLUSÕES}

A temperatura de calcinação tem fator preponderante na obtenção de ferritas dopadas com cobre e magnésio com propriedades absorvedoras de radiação eletromagnética. $\mathrm{O}$ efeito de difusão e crescimento das partículas inflencia no efeito de absorção porque partículas nanométricas não tem estrutura de domínios magnéticos que tem fator principal na absorção de radiação. Assim o pó calcinado a $900^{\circ} \mathrm{C}$ produziu o melhor resultado devido a dois fatores: a formação de multidomínios que caracteriza partículas maiores que 600 $\mathrm{nm}$ e a substituição de íons cobre em posições octaedrais com aumento de magnetização e aumento de absorção de radiação. $\mathrm{O}$ material sintetizado pode ser empregado na confecção de antenas de telefonia celular considerando que apresenta entre $40 \%$ e $77 \%$ de absorção de radiação eletromagnética.

\section{REFERÊNCIAS}

[1] M. V. Vasic, Zn, Ni Ferrite/NiO nanocomposite powder obtained from acetylacetonato complexes, Nanotechnology 17 (2006) 4877-4884.

[2] R. Simões, M. Rezende, E. L. Nohara, Materiais Absorvedores de Radiação Eletromagnética Baseados em Ferritas MnZn, Rev. Ci. Exatas 12 (2005) 121-124.

[3] E. E. Sileo, R. R. Rotelo, S. E. Jacobo, Nickel zinc ferrites prepared by the citrate precursor method, Physica B 320 (2002) 257-260.

[4] A. Thakur, M. Singh, Preparation and characterization of nanosize $\mathrm{Mn}_{0,4} \mathrm{Zn}_{0,6} \mathrm{Fe}_{2} \mathrm{O}_{4}$ ferrite by citrate precursor method, Ceram. Int. 29 (2003) 505-511.

[5] I. Z. Rahman, T. T. Ahmed, A study on Cu substituted chemically processed $\mathrm{Ni}-\mathrm{Zn}$-Cu ferrites, J. Magn. Magn. 
Mater. 290-291 (2005) 1576-1579.

[6] T. T. Ahmed, I. A. Rahman, S. A. M. Tofail, Effect of copper ion distribution on the magnetization of nanoscaled NiZn ferrite, J. Magn. Magn. Mater. 272-276 (2004) 22502252.

[7] U. R. Lima, M. C. Nasar, R. S. Nasar, M. C. Rezende, J.
H. Araújo, Ni-Zn nanoferrite for radar-absorbing material, J. Magn. Magn. Mater. 320 (2008) 1666-1670.

[8] U. R. Lima, M. C. Nasar, R. S. Nasar, M. C. Rezende, J. H. Araújo, J. F. Oliveira, Synthesis of NiCuZn ferrite nanoparticles and microwave absorption characterization, Mater. Sci. Eng. B 151 (2008) 238-242.

(Rec. 04/06/2012, Rev. 13/04/12, Ac. 07/05/12) 